\title{
Human genetic association studies: genomic association data and its discloser for public access, barrier to human privacy and protection
}

\begin{abstract}
The achievements of Human Genome Project and subsequent advancements in Genotyping have led to an influx of exciting new developments in genetics. Technology has provided scientists with a comprehensive data on human genomes as human genome is now capable to incite in depth and precise data information that allows access to detailed DNA sequences in order to analyze clinical questions. The methods employed have been optimized to examine the application of Genome Wide Association Studies with population based forensic investigations. Genome Wide Association Studies associated approaches have also been incorporated in routine clinical practice.
\end{abstract}

Keywords: human genome project, genome wide association studies, genome, gene, research design

\author{
Volume 5 Issue I - 2018 \\ Fahmida Khatoon,' Farhan Essa Abdullah, ${ }^{2}$ \\ Zahid Balouch ${ }^{3}$ \\ 'Department of Biochemistry, Faculty of Medicine, United \\ Medical College, Pakistan \\ 2Professor \& CEO, Department of Microbiology, Dr. Essa \\ laboratory and Diagnostic Centre, Pakistan \\ ${ }^{3}$ Assistant Professor, United Medical College, Pakistan
}

Correspondence: Fahmida Khatoon MBBS, MPhil,Associate Professor, Department of Biochemistry, United Medical College, Pakistan, Tel 50972078I,

Email fahmida.khatoon@yahoo.com,drfahmidal@gmail.com

Received: December 22, 2017| Published: February 02, 2018

\section{Introduction}

The use of Genome Wide Association Studies for detecting many diseases of forensic interest and traits for sex distributions are due to sex associated variations in prevalence. ${ }^{1}$ Genome Wide Genotypic Data from a person for the purpose of utilizing it for forensic sampling in Genome Wide Association Studies. This research further evaluates the actions taken by the authorities in USA to inhibit public access to Genome Wide Data dispersion but however, limitations have been observed to restrict sensitive information and also the use of genotype frequency data from each of the previous study which have been published using funding from public services agencies like National Institute of Health (NIH) and Welcome Trust. Reactions to this implication in the public sector has been not so encouraging when authorities consider it too late and too little that has been done in maintaining privacy for individuals. However, the response from responsible persons in genetic field of experimentation models declared the breaching of public data as a high level beaurocratic response to a minimum risk which can unnecessarily inhibit the futuristic scientific research. ${ }^{2}$ Other scientific concerns were also been highlighted concerning to situations when an individual's identity is accurately determined by applying Genome Wide Association Studies datasets. Several other researchers have also signified that misuse of an identification of an individual along with familial and medical history records and if genetics will not held these sensitive data information it will backfire to their own capabilities to carry further sequence of research network. Church notified that appropriateness of measures in restricting access to information is likely to exclude the scientists who are specialist in handling sophisticated data. ${ }^{3}$ The active researchers in the field of genomic wide studies further argue that consent for complete disclosure for releasing genomic information should be taken from the respondents rather than making this procedure difficult to access for promises for anonymity.
In similar context, Martin and Bobrow compared and contrasted the related potential risks and advantages in execution, hence proposed four stepped representation:

a. For current scenarios limited access to personal genetic information need to be implemented.

b. Declaration of any activity that comes under the category of illegalarity need to be banned or be termed as malicious practice.

c. Increase in the level of knowledge for the general public to identify the nature of their personal information that can be misused for purposes harmful for them in physical, social or financial frameworks.

d. Encouragement for appropriate recognition need to be stimulated in order to understand the professional relationship between the genetic researchers and the study participants.

The sex and ancestry related data and genetic information are important components for Quantity Check (QC) in GWAS and can be implemented in practice to remove misidentifications of samples and stratifications in population samples. The approach to identify the mis-identification in processes where strong associations are detected between genotype and phenotype characteristics can be inferred if the observed phenotype is expected to be caused by the observed genotype for every subject within the study. ${ }^{4}$ The study of genomic and associated genetic markers requires the step wise following of techniques like firstly, a set of information about genotype and phenotype relationship need to be established and identified. ${ }^{2}$ Secondly, phenotypes need to be ascertained at additional cost before the initiation of study, possibly for reasons of extracting usual phenotype data during the collection method. Thirdly, the modeling for the mixture sample that is under consideration for estimating phenotype and genotype relationship 
needs to be optimized to deliver highest set of information. Finally, the combination of all the information assessed from the relationships should be higher enough to give better sensitivity and specificity. It can also be possible to assess Single Nucleotide Polymorphisms (SNPs) in determining genotype for each sample, before the execution of costly Genome Associated Studies or Genome Sequencing into practice. $^{2}$

\section{Population genomics and access to genomic datasets}

Homer illustrated that Single Nucleotide Polymorphisms data from respondents inducted in Genome Association Studies can depict that individuals' genetic mixture consists of up to 1000 respondents whose DNA profiling have been disclosed for research proposes. As the mentioned case implies the basic statistical theory, the condition illustrated that this needs to elevate the frequency of subsequent discussions that can minimize the privacy concerns and trigger anticipating scientific possibilities (Human Genome Project Information, 2003). For example, there is a strong relationship between geographic correlation and genetics similarity of an individual who resides in Europe, but there is a considerable overlap of genetic similarity among neighboring European subpopulations which determines accurate determinations for genetic variability. ${ }^{5}$

\section{Phenotype extraction, incorporated into genomic studies and snps}

The genetic effect of Single Nucleotide Polymorphisms (SNPs) on the phenotype of an individual depends on the number of contributing SNPs and non-genetic influences like environmental effects, in determining accurately an identity of a person. Phenotype studies illustrate that eye color is the most successfully predictable phenotype essential in accurately identifying basic physical factors in an individual. ${ }^{5}$ The challenges undertaken in applying DNA prediction into categorizing appearance traits and eye color is its expected variability in conceptual understanding of trait information. For example, people assign same eye color to various color categories and therefore look different from others using an eye color provided by DNA predictions. In order to minimize this problem, studies investigated about the genetic basis of variation in eye color utilizing SNP data analysis. ${ }^{6,7}$

\section{Genetic mapping scheme in analyzing familial linkages}

In order to demonstrate the heritable characteristics of an unidentified person and to detect mutations at the loci implies that Genetic Mapping Scheme is based on linkages in familial studies of randomly illustrated phenotypical characteristics. However, it is difficult to ascertain that as to how polymorphic loci are adhered to establish linkage relationship into practice. To address such problems, to adapt linkages to a polymorphic marker is better than the availability of no linkage. ${ }^{2}$

\section{Structured association and genetic markers in gwas}

The technique of Structured Association works on the principle of Hardy and Weinberg's Equilibrium within populations with linkages to genetic markers that are also responsible for establishing equilibrium to detect and correct stratification in a homogenous sub group cluster based on genotypic characteristics. Test for detecting familial or hereditary pathological traits in the generations can be achieved by studying association of each genetic marker to disease expression phenotype. ${ }^{8-10}$

\section{Failures of linkage for complex disease}

Cystic fibrosis (and most rare genetic disorders) can be caused by multiple different genetic variants within a single gene. Because the effect of the genetic variants is so strong, cystic fibrosis follows an autosomal dominant inheritance pattern in families with the disorder. One of the major successes of human genetics was the identification of multiple mutations in the CFTR gene as the cause of cystic fibrosis. ${ }^{11}$ This was achieved by genotyping families affected by cystic fibrosis using a collection of genetic markers across the genome, and examining how those genetic markers segregate with the disease across multiple families. This technique, called linkage analysis, was subsequently applied successfully to identify genetic variants that contribute to rare disorders like Huntington disease. ${ }^{12,13}$

\section{Conclusion}

Over the span of last 10years, the fields of genomics have under taken interesting and dramatic shifts in how to comprehend forensic detailing with respect to investigations about unknown pedigree, unidentified complex diseases and ethnogeographic ancestry of subjects as studied by Scott, in $2007 .{ }^{10}$ The wealth of information which can be extracted from immense map of human genome along with advancements in genotyping and related technologies has presented many opportunities for the scientists to test assumptions about the complex genomics for human. This has led a new era of genetic investigation like Genomic Association studies that incorporates to solve analytical questions about heritage and population based studies.

\section{Future direction}

Genome-wide association studies have identified new genetic risk factors for many common human diseases and have forced the genetics community to think on a genome-wide scale. Looking towards the future, it seems evident that the current enhancements in Genome Wide Association Studies is going to transform through changes for finding genetic susceptibilities that are responsible for causing characteristics unique to a familial trait. In 2010, Durbin provided a series of projects in the ' 1000 Genomic Project' that has provided an avenue for testing assumptions by utilizing sequencing techniques to identify rare variations in human genome. The use of sequential data for detecting rare genes for variant determination has also been observed possible through association analysis.

\section{Acknowledgements}

None.

\section{Conflict of interest}

Author declares that there is no conflict of interest.

\section{References}

1. Distefano JK, Taverna DM. Technological issues and experimental design of gene association studies. Methods Mol Biol. 2011;700:3-16.

2. Al Safar HS, Abidi FH, Khazanehdari KA, et al. Evaluation of different sources of DNA for use in genome wide studies and forensic application. Appl Microbiol Biotechnol. 2011;89(3):807-815.

3. Alexander DH, Novembre J, Lange K. Fast Model-Based Estimation of Ancestry in Unrelated Individuals. Genome Res. 2009;19(9):1655-1664. 
4. Hindorff L, Junkins $\mathrm{H}$, Hall $\mathrm{P}$, et al. A catalog of published genome-wide association studies. 2009.

5. Jakobsson M, Scholz SW, Scheet P, et al. Genotype, haplotype and copy number variation in worldwide human populations. Nature. 2008;451(7181):998-1003.

6. Johnson AD, Leslie R, O Donnell CJ. Temporal Trends in Results Availability from Genome-Wide Association Studies. PLoS Genet. 2011;7(9):e1002269.

7. Mangold E, Ludwig KU, Birnbaum S, et al. Genome wide association study identifies two susceptibility loci for nonsyndromic cleft lip with or without cleft palate. Nature Genet. 2010;42(1):24-26.

8. Abecasis GR, Altshuler D, Auton A, et al. A Map of Human Genome Variation from Population-Scale Sequencing. Nature. 2010;467(7319):1061-1073.
9. Kerem B, Rommens JM, Buchanan JA, et al. Identification of the cystic fibrosis gene: genetic analysis. Science. 1989;245(4922):1073-1080.

10. MacDonald ME, Novelletto A, Lin C, et al. The Huntington's disease candidate region exhibits many different haplo- types. Nat Genet 1992;1(2):99-103.

11. Scott RC, Juhász G, Neufeld TP. Direct induction of autophagy by Atg1 inhibits cell growth and induces apoptotic cell death. Curr Biol. 2007; 17(1):1-11.

12. Bird TD. Genetic aspects of Alzheimer's Disease. Genet Med. 2008;10(4):231-239

13. Kayser M, Liu F, Janssens AC, et al. Three Genome-wide Association Studies and a Linkage Analysis Identify HERC2 as a Human Iris Color Gene. Am J Hum Genet. 2008;82(2):411-423. 